\title{
A demonstration of discriminative conditioning in the blow fly, Phormia regina
}

\author{
JEFFRY P. RICKER, JOHN N. BRZORAD, and JERRY HIRSCH \\ University of Illinois at Urbana-Champaign, Champaign, Illinois
}

\begin{abstract}
We present conclusive evidence for the classical conditioning of Phormia regina (the blow fly) by performing necessary control procedures that have been lacking in previous studies. Experiment 1 replicated successfully the study by Zawistowski and Hirsch (1984) which showed (1) that flies receiving a discriminative conditioning procedure responded (by extending their proboscises) differentially to a CS+ and CS- and (2) that flies receiving a random control procedure did not respond differentially. Experiment 2 controlled for experimenter bias by using an automatic stimulating procedure and performing blind tests. The two experiments together provide the most fully controlled evidence for learning in blow flies.
\end{abstract}

The study of conditioning in flies has been hampered by use of inadequate control procedures and the neglect of contradictory results (Holliday \& Hirsch, in press; Ricker, Hirsch, Holliday, \& Vargo, 1986). We consider the contingency between a conditioned stimulus (CS) and an unconditioned stimulus (US) to be the parameter important for the occurrence of learning in a classical conditioning procedure (LoLordo, 1979; Rescorla, 1967). Therefore, if one is to infer learning, then the contingency between CS and US must be varied while controlling for all other (nonassociative) variables. In addition, the effects of specific situational variables must be controlled, such as conditions associated with a given testing situation and systematic experimenter (E) bias. All past studies of Phormia regina have failed, at least in part, to control adequately for nonassociative variables in their conditioning procedures (see Ricker et al. for a detailed discussion).

In the present study we attempted to demonstrate conclusively classical conditioning in $P$. regina. In Experiment 1 , the contingency between CS and US was varied. This experiment replicated one performed by Zawistowski and Hirsch (1984). To control for the effects of $E$ bias, in Experiment 2 we studied, with a blind testing procedure, the effects on performance of differences in contingencies.

\section{EXPERIMENT 1}

Zawistowski and Hirsch (1984) described a procedure, based on one developed by Nelson (1971) and McGuire and Hirsch (1977), for discriminative conditioning of the

This work was supported by Grant BNS-83-00353 from the National Science Foundation and by Grant MH 15173 for training in research on institutional racism from the National Institute of Mental Health. The primary data for this paper are available in the thesis submitted by the second author to the University of Illinois at Urbana-Champaign (Brzorad, 1985) in partial fulfillment of the requirements for the degree of Master of Arts. Send requests for reprints to J. Hirsch, Department of Psychology, 603 E. Daniel Street, Champaign IL 61820. proboscis extension. In this procedure, contingencies between CS and US are varied in two ways: first, by pairing one $\mathrm{CS}(\mathrm{CS}+$ ) with the US (resulting in a contingency of 1.0) and presenting another $\mathrm{CS}(\mathrm{CS}-)$ unpaired with the US (resulting in a negative contingency); second, by reversing, in a different test session and with the same fly, the CS with which the US is paired (i.e., the original CS+ becomes the new CS-, and vice versa).

\section{Method}

Subjects. Two laboratory lines were crossed to found the SYN line (see Zawistowski \& Hirsch, 1984). Subjects for this experiment were sampled 45, 46, and 48 generations after the initial cross.

Pretest procedure. See Zawistowski and Hirsch (1984) for details on husbandry. On the first full day of eclosion, flies were chill anesthetized, separated by sex, and placed in $30.5 \times 30.5 \times 30.5 \mathrm{~cm}$ insect cages. Sucrose and water were provided ad lib for $24 \mathrm{~h}$, after which time flies were kept individually in glass specimen bottles containing only watersoaked cotton. Two days later, flies were placed in plastic pipette tips with the head and forelegs protruding from the narrow end (illustrated in Tully, Zawistowski, \& Hirsch, 1982), allowed to rest $1 \mathrm{~h}$, satiated with water (as in Zawistowski \& Hirsch), and placed in a testing rack.

Test. Four groups of 5 flies (a total of 20 flies, with equal numbers of males and females) were sampled for a given testing session. The four groups consisted of two experimental groups ( $1 \mathrm{M} \mathrm{KCl}$ used as CS+ and $1 \mathrm{M} \mathrm{NaCl}$ used as $\mathrm{CS}-$, and vice versa) and two "random" control groups (see below). Two samples from the SYN line were tested; the first came from generations 45 and 46 and the second from generation 48.

A testing session consisted of 30 trials ( 15 presentations of each CS). Each trial was separated by a 5-min intertrial interval. The CSs, contained in test tubes, were presented to the fly by immersing the tarsi for $5 \mathrm{sec}$ each. The US solution was touched to the labellum on the last second of CS presentation. A session was divided into three blocks of 10 trials each. A difference score was calculated for each fly in each block by subtracting the number of responses to the $\mathrm{CS}-$ from the number of responses to the CS+.

Testing occurred over a 2-day period. On the first day (the test), the sequence of CS+ and CS- presentations was randomized, allowing no more than three consecutive presentations of one stimulus. At the end of this session, each fly was placed back into a bottle containing watersoaked cotton. On the next day (the reversal), flies were water satiated and the sequence of CSs was reversed.

On all three blocks of trials, the random control groups received the same order of CS presentations as did the experimental groups. However, 
the US was presented so that each CS was paired with the US five times in each block (i.e., there was a 0.5 contingency with each CS). This procedure differed from that of Zawistowski and Hirsch (1984); in the latter study, the control group was given the discriminative conditioning procedure on the last block of trials.

\section{Results}

Flies were discarded that did not respond to the US on at least 12 trials in either test session (5 and 3 from the experimental and control groups, respectively). Six flies escaped (5 and 1 from the experimental and control groups, respectively). To equalize sample sizes to 30 in each treatment, 6 flies were discarded randomly from the control group. A mixed-design two-way ANOVA with repeated measures was used to analyze the data. This statistical model assumes homogeneity of within-treatment variances; thus, these were tested with a Levene test (Keppel, 1973, pp. 81-83). This analysis showed that variances were homogeneous among blocks for the experimental group but not for the control group $(p<.05)$. Therefore, the data were transformed by using arcsin $\sqrt{X+0.5 / N+1}$, where $X$ represents the difference score of an individual and $N=5$, the number of presentations of each CS in a block of trials (Tully, 1981/1982). A Levene test again showed that the variances were homogeneous for the experimental group but not for the control group. Therefore, untransformed scores were used in the data analysis. Keppel noted that heterogeneity will bias the calculated $F$ ratio positively when the repeated factor is being considered.

Means of difference scores and their $95 \%$ confidence intervals are plotted in Figure 1. The summary table for the ANOVA is presented in Table 1 . There were significant main effects for both the group and block factors, indicating that the mean difference scores of the experimental and control groups differed from each other, as did those of blocks within groups. There was no sig-

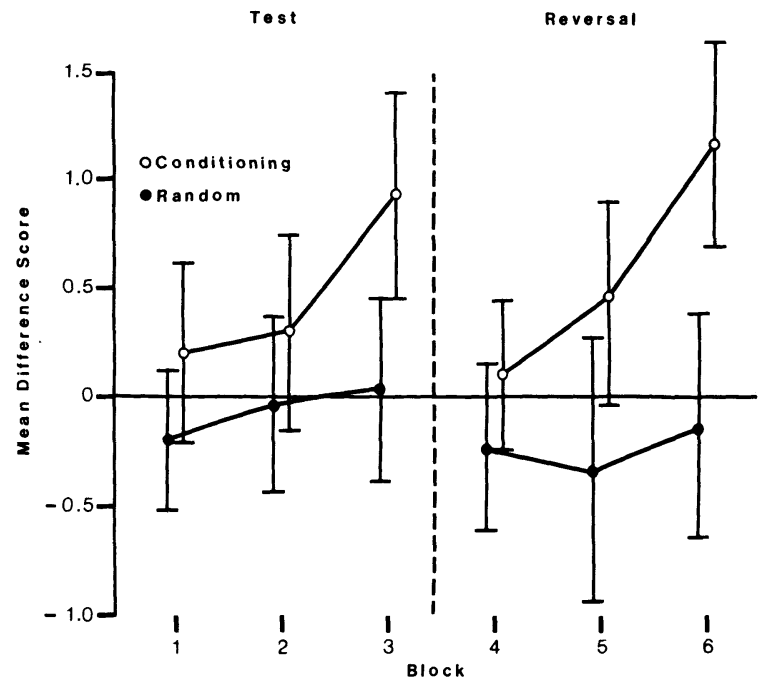

Figure 1. Mean difference scores over blocks on the test and reversal sessions for flies receiving either the discriminative conditioning or random control procedures.
Table 1

Summary Table for Two-Way ANOVA with Repeated MeasuresRandom Control Experiment

\begin{tabular}{lrrrr}
\hline \multicolumn{1}{c}{ Source } & df & \multicolumn{1}{c}{ SS } & \multicolumn{1}{c}{ MS } & F \\
\hline Group & 1 & 45.51 & 45.51 & $22.07^{*}$ \\
Subject/Group & 58 & 119.61 & 2.06 & \\
Block & 5 & 23.62 & 4.72 & $3.77^{*}$ \\
Group $\times$ Block & 5 & 4.92 & 0.98 & 0.79 \\
Block $\times$ Subject/Group & 290 & 363.12 & 1.25 & \\
\hline
\end{tabular}

${ }^{*} p<.05$.

nificant interaction between groups and blocks, which suggests that the changes over blocks in mean difference scores were similar for the two treatments. However, Figure 1 shows that, whereas the experimental group differs significantly from zero on the last block of trials in both the test and reversal sessions, the control group does not do so in any block. Therefore, differential conditioning to the two CSs occurred in the experimental group but not in the control group.

The reversal (Blocks 4 through 6) shows that a change in contingency will result in changes in acquisition: The mean difference score of the experimental group increased over blocks, indicating that when the original CS- is put in the position of the CS+, it will be responded to with greater frequency than the original $\mathrm{CS}+$, when the latter is placed in the position of the CS-. Additionally, if memory of the contingencies in the first session were present when the second session was begun, then one would expect that the mean difference score for the experimental group on Block 4 would be significantly lower than on Block 1. However, Figure 1 presents no evidence for memory: The mean difference score on Block 4 is not lower than that on Block 1.

\section{Discussion}

The results of this experiment replicate those of Zawistowski and Hirsch (1984) with one exception: The latter study reported a significant interaction between groups and blocks. However, because the mean difference score of the experimental group differed significantly from zero on the last block of trials in both sessions, and because that of the control group did not so differ, the present study has been successful in replicating the conditioning effects of Zawistowski and Hirsch.

\section{EXPERIMENT 2}

Because of the possibility of $\mathrm{E}$ bias, the results of Experiment 1 are not conclusive evidence for a learning interpretation. Differences have been found among investigators in the results obtained with this technique (Ricker et al., 1986). Therefore, E bias was excluded in two ways: by a procedural change so that the stimuli were presented automatically, and by the use of blind testing.

\section{Method}

Subjects. Flies were sampled from generation 52 of the SYN line. Pretest procedures. Flies were treated as in Experiment 1 with one exception: Because no reversal was performed, they were not placed into individual bottles but, instead, were left in the insect cages. As in Experiment 1, sucrose was provided for the first $24 \mathrm{~h}$. 
Test. Vargo, Holliday, and Hirsch (1983) modified a technique developed by Médioni and Vaysse (1975) for stimulating D. melanogaster automatically. A kymograph drum is used, across which flies can walk; strips of filter paper are placed obliquely along the drum. Plastic containers, filled with the CS and US solutions, are placed at the top of the drum; glass capillary tubes descend from these containers to the strips of paper. Different strips are moistened with different stimuli and are placed on the drum so that, as the drum rotates, flies will experience the different stimuli at appropriate time intervals.

Zawistowski and Hirsch (1984) adapted this technique for testing $P$. regina with a discriminative conditioning procedure. The drum was set to rotate once every $5 \mathrm{~min}$. At this speed, a fly experiences each CS for about $5 \mathrm{sec}$ and the US for about $2.5 \mathrm{sec}$ (because a fly might move its legs, CS and US stimulations could range from 2 to $6 \mathrm{sec}$ and from 1 to $3 \mathrm{sec}$, respectively). The CS+ and US strips were separated by a rubber partition that allowed a trace of about $1 \mathrm{sec}$. The CS- strip was placed so that it was presented $2.5 \mathrm{~min}$ after the preceding US stimulation and $2.5 \mathrm{~min}$ before the succeeding CS+ stimulation

Two treatments were tested, with the particular treatment being unknown to $\mathrm{E}$. In the experimental treatment, flies were presented with a $\mathrm{CS}+$ and a CS - that were different salt solutions (i.e., CS+ was $1 \mathrm{M} \mathrm{KCl}$ and CS- was $1 \mathrm{M} \mathrm{NaCl}$, or vice versa). In the control treatment, the same salt solution was presented in both CS positions (i.e., both $\mathrm{CS}+$ and $\mathrm{CS}-$ were either $1 \mathrm{M} \mathrm{KCl}$ or $1 \mathrm{M} \mathrm{NaCl}$ ). The former treatment presents a contingency of 1.0 between CS+ and US, whereas the latter presents one of 0.5 . Sixty flies were tested in each of the four possible stimulus situations so that a total of 120 flies were given each treatment. Each CS was presented 15 times.

\section{Results}

If flies did not participate (i.e., did not walk over the strips of paper) on three or more CS+ or CS- presentations, they were excluded from the analysis. Thus, we discarded $20(17 \%)$ and $29(25 \%)$ flies from the experimental and control groups, respectively; another 5 and 6 from the experimental and control groups, respectively, were discarded for responding to sucrose fewer than 12 times. Two escaped from the experimental group, leaving 93 and 85 flies in the experimental and control groups, respectively. To equalize sample sizes, 8 flies were discarded randomly from the experimental group. The statistical model for the ANOVA was the same as in Experiment 1. A Levene test showed that the withintreatment variances were homogeneous for both the experimental and control groups $(p>.05)$.

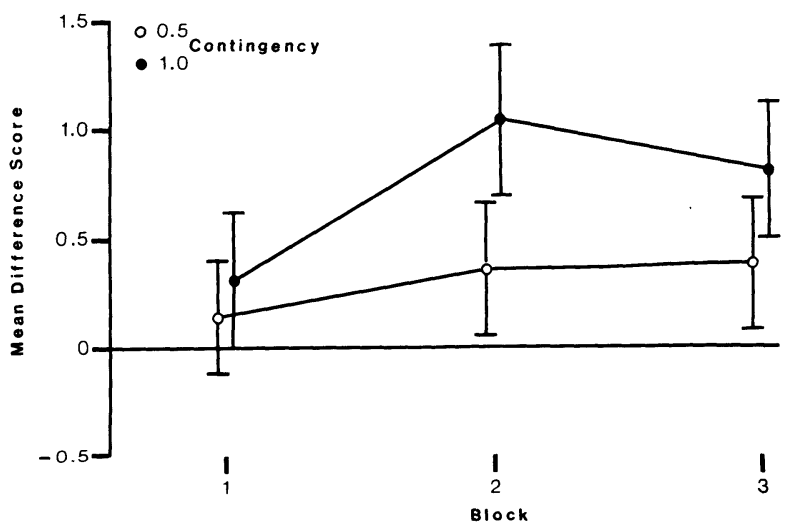

Figure 2. Mean difference scores over blocks for flies receiving a 0.5 or a 1.0 contingency between $\mathrm{CS}+$ and US.
Table 2

Summary Table for Two-Way ANOVA with Repeated MeasuresContingency Experiment

\begin{tabular}{lrrrl}
\hline \multicolumn{1}{c}{ Source } & df & \multicolumn{1}{c}{ SS } & \multicolumn{1}{c}{ MS } & F \\
\hline Group & 1 & 22.87 & 22.87 & $6.19^{*}$ \\
Subject/Group & 168 & 620.61 & 3.69 & \\
Block & 2 & 21.28 & 10.64 & $9.53^{*}$ \\
Group $\times$ Block & 2 & 5.45 & 2.72 & 2.44 \\
Block $\times$ Subject/Group & 336 & 375.27 & 1.12 & \\
\hline
\end{tabular}

${ }^{*} p<.05$.

The means of difference scores and their $95 \%$ confidence intervals for both groups over the three blocks are plotted in Figure 2. Table 2 presents a summary of the ANOVA. Both main effects are statistically significant, which indicates that the amount of learning differed between the two groups. It is evident in Figure 2 that both groups showed increases in the mean difference score over blocks, but that the group experiencing a 1.0 contingency reached a higher level than did the group experiencing a 0.5 contingency.

\section{Discussion}

Systematic E bias cannot explain the differences in performance between the experimental and control groups. Therefore, the difference in average performance between them must be due to differences in the amount of learning by individuals of the two groups. The present study provides the most fully controlled evidence to date for learning in $P$. regina.

\section{REFERENCES}

Brzorad, J. N. (1985). Conditioned discrimination in Phormia regina. Unpublished master's thesis, University of Illinois, UrbanaChampaign.

Holliday, M. J., \& HirsCh, J. (in press). A comment on the evidence for learning in Diptera. Behavior Genetics.

KePPEL, G. (1973). Design and analysis: A researcher's handbook. Englewood Cliffs, NJ: Prentice-Hall.

LoLordo, V. (1979). Classical conditioning: Contingency and contiguity. In M. Bitterman, V. LoLordo, J. Overmier, \& M. Rashotte (Eds.), Animal Learning: Survey and Analysis (pp. 61-97). New York: Plenum Press.

MCGuiRE, T. R., \& HiRSCH, J. (1977). Behavior-genetic analysis of Phormia regina: Conditioning, reliable individual differences, and selection. Proceedings of the National Academy of Sciences of the United States of America, 74, 5193-5197.

MÉdIONI, J., \& VAYSSE, G. (1975). Suppression conditionelle d'un réflexe chez la Drosophile (Drosophila melanogaster): Acquisition et extinction. Comptes Rendus des Séances de la Société de Biologie, 169, 1386-1391.

Nelson, M. C. (1971). Classical conditioning in the blowfly (Phormia regina): Associative and excitatory factors. Journal of Comparative \& Physiological Psychology, 77, 353-368.

RESCORLA, R. A. (1967). Pavlovian conditioning and its proper control procedures. Psychological Review, 74, 71-80.

Ricker, J. P., Hirsch, J., Holliday, M. J., \& VARGo, M. A. (1986). An examination of claims for classical conditioning as a phenotype in the genetic analysis of Diptera. In J. L. Fuller \& E. C. Simmel (Eds.), Perspectives in Behavior Genetics (Vol. 2). Hillsdale, NJ: Erlbaum.

TULLY, T. P. (1982). Behavior-genetic analysis of the black blow fly, Phormia regina, using the central excitatory state (CES) (Doctoral dissertation, University of Illinois, 1981). Dissertation Abstracts International, 42, 3568B. 
Tully, T., Zawistowski, S., \& HiRsch, J. (1982). Behavior-genetic analysis of Phormia regina: III. A phenotypic correlation between the central excitatory state (CES) and conditioning remains in replicated $\mathrm{F}_{2}$ generations of hybrid crosses. Behavior Genetics, 12, 181-191.

VarGo, M., Holliday, M., \& Hirsch, J. (1983). Automatic stimulus presentation for the proboscis extension reflex in Diptera. Behavior Research Methods \& Instrumentation, 15, 1-4.

ZAWISTOWSKI, S., \& HIRSCH, J. (1984). Conditioned discrimination in the blow fly, Phormia regina: Controls and bidirectional selection. Animal Learning \& Behavior, 12, 402-408.

(Manuscript received for publication January 17, 1986.) 